\title{
Research on Smart Park Information System Design Based on Wireless Internet of Things
}

\author{
https://doi.org/10.3991/ijoe.v13i05.7055 \\ Meiyan Du \\ Department of General Education, Shandong University of Arts, Shandong, China \\ gbothj589153@126.com
}

\begin{abstract}
The purpose is to construct smart park platform by using the Internet of Things technology, to realize the access of intelligent systems and equipment within park, and to effectively coordinate the various systems in the park. Taking advantage of the key technologies of Internet of Things and cloud computing, smart park information hardware architecture and technical solutions are put forward, the sensor and wireless Internet of Things are applied to realize the system. The experimental results showed that the system realizes the information sharing, exchange and fusion between the various sensing subsystems, and solves the information islands phenomenon that existed in the past. In conclusion, this system can meet the actual needs of smart park, and provides a certain reference for similar park construction.
\end{abstract}

Keywords - Internet of Things, cloud computing, smart park

\section{Introduction}

At the present, the construction and development of China's smart city is closely related to all walks of life. The construction of smart park refers to concept and theory of smart city to a large extent, it is a regional epitome, and its development model can effectively promote smart city's construction and development [1]. This paper summarizes and concludes the high-quality ideas and detours of smart park development, rapidly and timely revises development program and ideas of smart park, thereby effectively promote the development of smart city. In present, the Internet of Things technology is still in the initial stage, a variety of technologies have to be developed and improved. The technology and level of information processing analysis determine the intelligent processing level of Internet of Things to a large extent. Cloud computing and intelligent identification are the mainly advanced technology [2]. Among them, the status of cloud computing is particularly significant; it is the core of the Internet of Things. It effectively promotes the integration IoT and Internet, and improves the realization of intelligence earth.

The purpose of smart park construction is multifaceted, but mainly through the construction of smart park to solve the problems like poor communication, duplication resources and information island, to achieve business and park win-win goal [3]. Therefore, the purpose of this study is to study and propose an effective solution to 
the information island based on the information and intelligent technology. Through the intelligent construction of environmental monitoring, transportation, security and information fusion to provide a reliable basic hardware and software, a variety of shared resources, cost-effective services and management.

\section{State of the art}

At this stage, the construction and development of smart city is quite hot in our country and it influences all walks of life. Smart park is a significant component and epitome of smart city, and its promotion and development model has a great role in promoting smart city. By discovering the advantages and disadvantages of the development and construction of the smart park, we can timely revise the development strategy of the smart city to avoid the detours of smart city process, and enhance the efficiency of smart city development process.

Smart park solutions are based on cloud computing, Internet of Things, and operations support. They are able to quickly and easily create, interconnect and organically combine the applications for various SaaS applications and Internet of Things [4]. For the park infrastructure to provide a new generation of green cloud computing data center and broadband bearer, access and indoor coverage network, build the basic network platform, provide seamless data access. Through the park service platform to provide various services business applications for the park settled enterprises, and provide operational management business applications for the park operations management units through the park management platform.

Smart park, which has "perception" or "thought", can fully collect the parks' of real-time information of relevant departments, location. It also provides efficient and timely comprehensive information and aid decision for individuals through intelligent processing. Smart park has four characteristics including demand as driving force, allround perception, internal-external development and multi-party collaboration. In the design and construction of smart park, it is necessary to strictly follow the stability, security, scalability, flexible information technology infrastructure, resource allocation deployment automation, multi-type billing service mode, comprehensive resource monitoring and emergency management, openness and other principles [5].

Based on the characteristics and construction principle of smart park, smart park is not equivalent to the previous wireless city and digital city, it's one of the new form city and it integrates "wisdom" and "smart". Therefore, successful smart park must have four major characteristics, including innovation, interconnection, multi-party integration, service management.

In recent years, modern science and technology has undergone tremendous changes, especially in the computer network technology, information technology, automatic intelligent control technology and Internet of things and so on. With the development of digital and Web technology, the future development trend of the social construction industry is bound to be more digital and intelligent.

Tang Shaolin designs and constructs the information platform of intelligent logistics park based on Internet of Things technology. The platform can track, monitor and 
manage the vehicles in the park through the Internet of Things technology, which greatly improves the efficiency of logistics and reduces the cost of logistics.

Based on the smart grid architecture of the intelligent campus infrastructure network, Miao Jiabo and Qiu Suchuan put forward the construction of smart grid to promote the park telecommunications network, the Internet, radio and television networks, networking and other multi-network integration. It not only reduces the cost of infrastructure construction, but also improves the construction efficiency. This will effectively promote the wisdom of the park construction.

Based on the analysis of the demand for the construction of the wisdom park, $\mathrm{Xu}$ Yanyan studies the Internet of Things era wisdom park construction program, including the use of Internet of Things technology platform for the construction of the wisdom of the park, intelligent park intelligent system access platform ideas and access methods. In the end, she completed the construction of intelligent platform based on M2M technology platform architecture and passed the test.

In summary, the above research not only reduces the cost of infrastructure construction, but also improves the efficiency of construction. However, these infrastructures are not smart enough. Therefore, we construct smart park platform by using the Internet of Things technology, to realize the access of intelligent systems and equipment within park, and to effectively coordinate the various systems in the park. Taking advantage of the key technologies of Internet of Things and cloud computing, smart park information hardware architecture and technical solutions are put forward, the sensor and wireless Internet of Things are applied to realize the system.

\section{$3 \quad$ Methods}

\subsection{System hardware design}

Smart park information system architecture is based on the ITU (International Telecommunication Union) architecture. The Internet of Things technical framework includes the perception layer technology, network layer technology, and application layer technology. System architecture is based on the perception, network, application three-layer structure, to achieve interconnection and resource sharing in a uniform standard. Through a variety of types of sensor deployment and setup, quickly and multi-directional precept park's information, through the transmission network send data to application system, and generate relevant information through the application system for display and processing.

Based on the actual information needs of park and system function, smart park information system generally includes environmental intelligent monitoring, intelligent transportation, intelligent security, intelligent integration processing, public information publishing services, and geographic information systems [6]. This paper focuses on the design of intelligent security modules and intelligent fusion processing modules.

Intelligent security adopts video surveillance to monitor the road, buildings and factories in the whole day surrounding the park. By displaying the distribution of all 
the video resources intuitively on the GIS map, operator can quickly choose and open the video image with mouse, in order to achieve video control based on GIS map. Based on the analysis of an abundant of video behavior, the utilization and reliability of system are improved. In this paper, intelligent security is based on the video control, we take advantage of cloud storage platform characteristic of massive storage platform and comprehensive analysis, comprehensively utilize video resources to achieve the park intelligent analysis, including staff-intensive alarm, boundary illegal stay warning, and suspicious items warning in specific areas [7 8]. The diagram is shown in Figure 1.

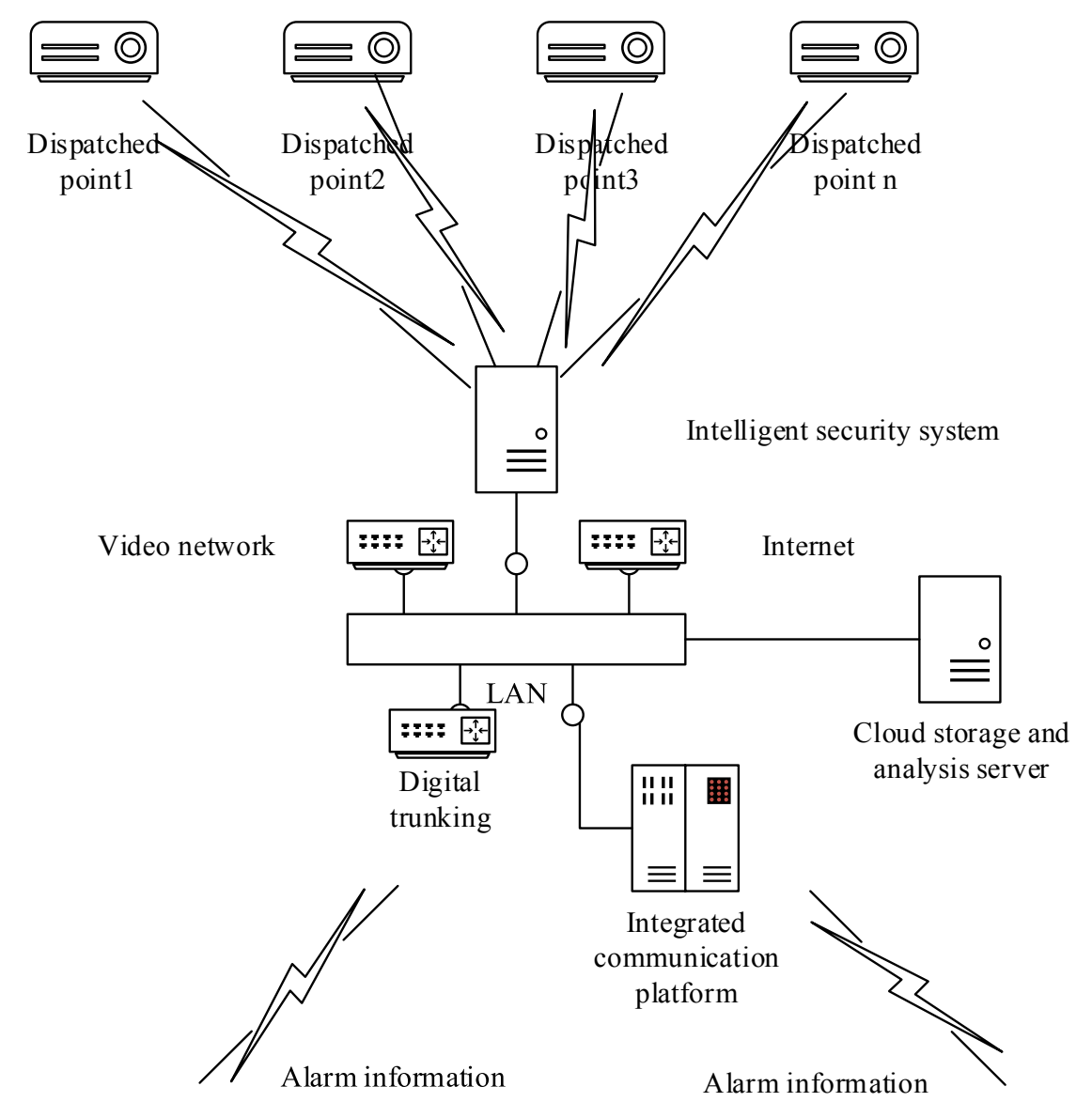

Fig. 1. Intelligent security system diagram

The main function of the intelligent fusion processing module is to receive and fuse the alarm information (such as toxic gas leakage alarm) from the intelligent environment monitoring system, to get the traffic accident sent by intelligent transportation system, to obtain illegal intrusion information sent by intelligent security system. 
After the communication with park security personnel is finished, appropriate measures will be taken. In this paper, we set the intelligent integration system of park management center, and realize multi-angle and multi-directional information fusion in order to make the information generated more comprehensive and timely. Through the interconnection with the park unit and the municipal police system, the hospital and other departments, we will quickly deal with emergencies for achieving rapid and effective the accident handing, and reduce harm of emergencies, solve the information island phenomenon formed in sub-system. The diagram is shown in Figure 2.

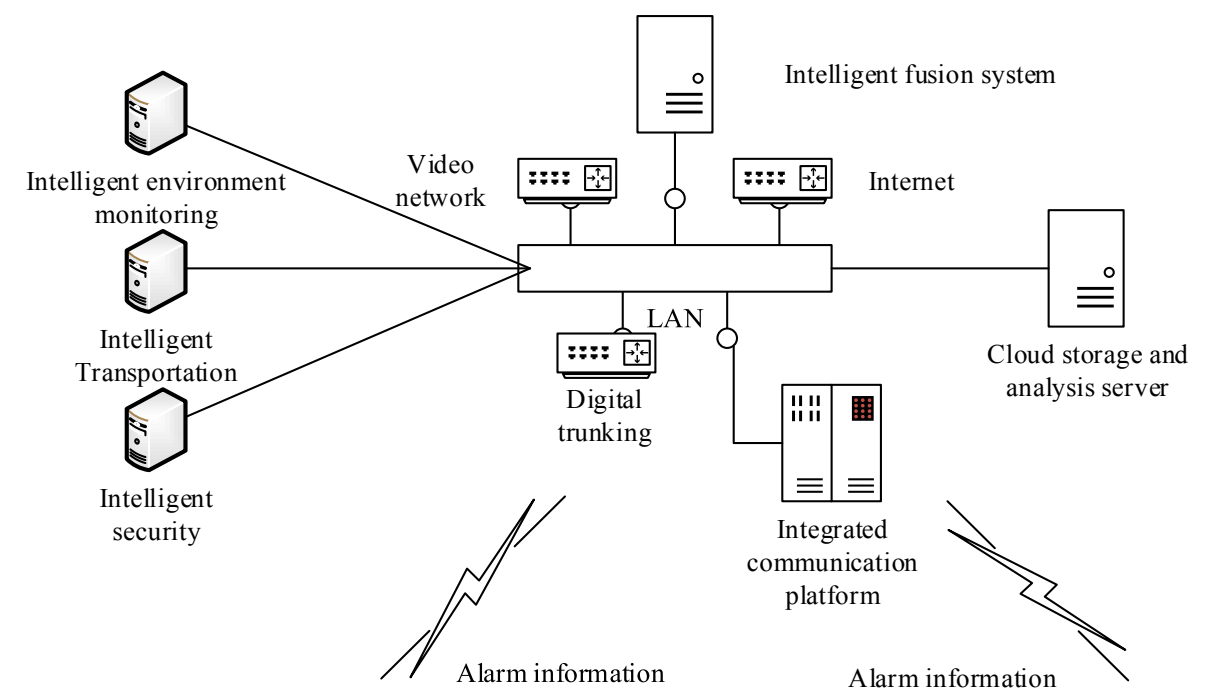

Fig. 2. Intelligent fusion diagram

\subsection{System software design}

Data collection is indispensable in the construction of smart park, only the realtime data collection could obtain timely decisions. The current data acquisition and processing functions are simpler. For example, we can use smoke sensor to monitor the toxic gases in the environment, and use temperature sensor to monitor the ambient temperature. When the smoke sensor detects the toxic gas, it will trigger the wireless transmission module and sends the alarm information to the data receiving processing part. And temperature sensor will send the real-time ambient temperature and other information through the wireless transmission module to the data receiving processing part. The processing flow is shown in Figure 3.

In the intelligent security module, the cloud computing and cloud storage platform can be used as the basis, to effectively offer the support to the large video surveillance system within park, including: various types of sensor access, video data storage and forwarding, intelligent analysis and processing, high-speed retrieval, etc. Cloud computing information system can make the massive data service quick requests possible. Through introducing SOA, we could build video resource application architecture 


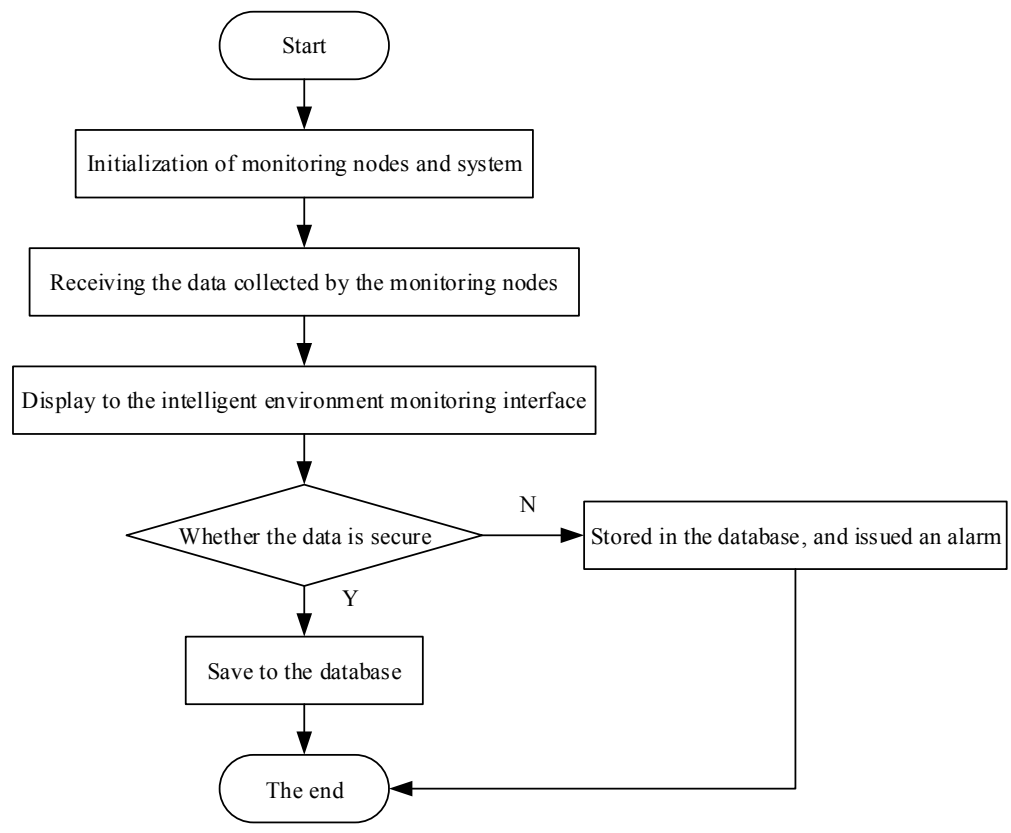

Fig. 3. Data acquisition and processing flow chart

based on service, meet the user demand from units, departments, individual within park, which have different levels and numbers, and improve the massive resources of the database high-speed retrieval capabilities. This system has high reliability, in the process of large-capacity video forwarding, storage and processing, it can realizes several functions, including electronic patrol, abnormal behavior and items monitoring, and key areas monitoring. Electronic patrol function is based on video surveillance network system, which greatly solves the shortcomings of traditional patrol system. The park security personnel can complete the key road monitoring work not in the field. Through the function, the personnel can find the suspicious "target" in time, and deal with it in advance, arrange other personnel who in field rushed to the scene, in order to greatly improve the speed of event handling. The software flow is shown in Figure 4.

Through the effective integration of intelligent environment monitoring system, intelligent transportation system, and intelligent security system, we can intelligently identify the same event, tackle its information island phenomenon based on preset judging principle, and achieve comprehensive display of park information. The control center receives the information of intelligent environment monitoring system, intelligent transportation system, and intelligent security system, etc. We also can identify the same event based on preset judging principle, and obtain fusion result and auxiliary decision-making plan (need manual confirmation), and then tackle the same event. Intelligent fusion processing flow chart is shown in Figure 5. 


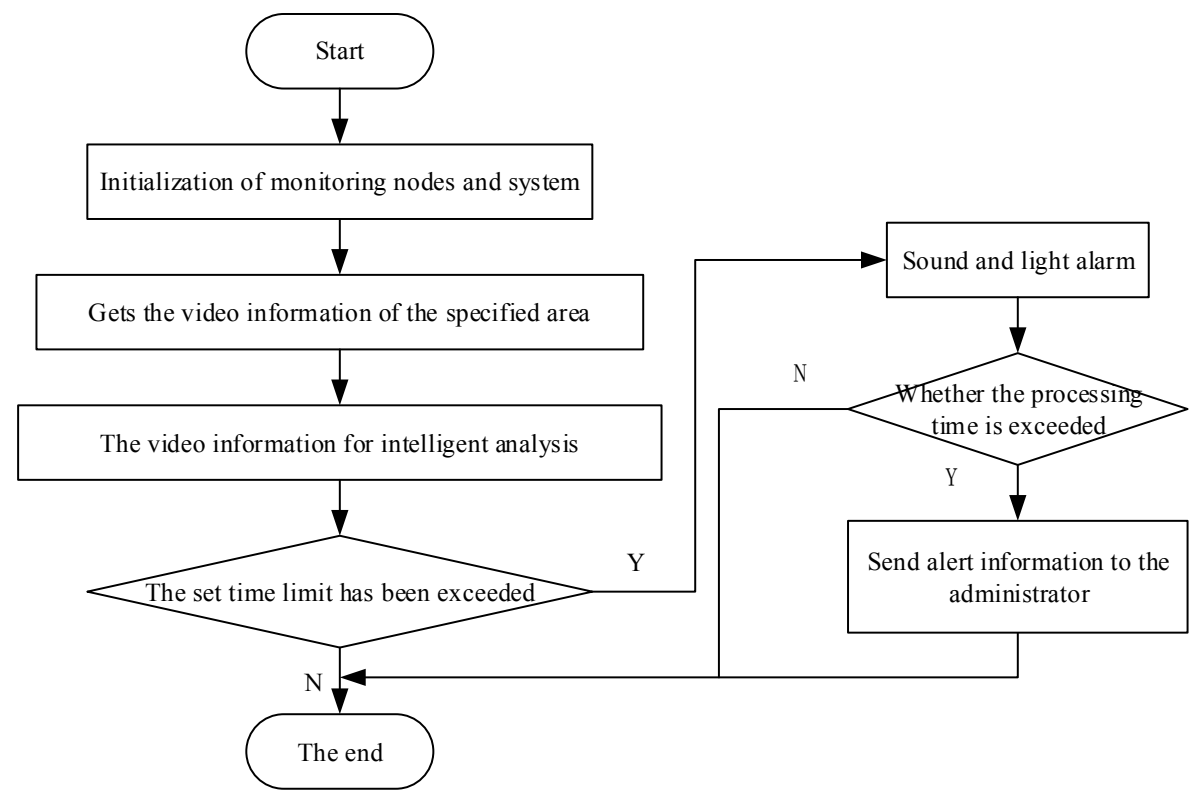

Fig. 4. Software flow chart of electronic patrol function

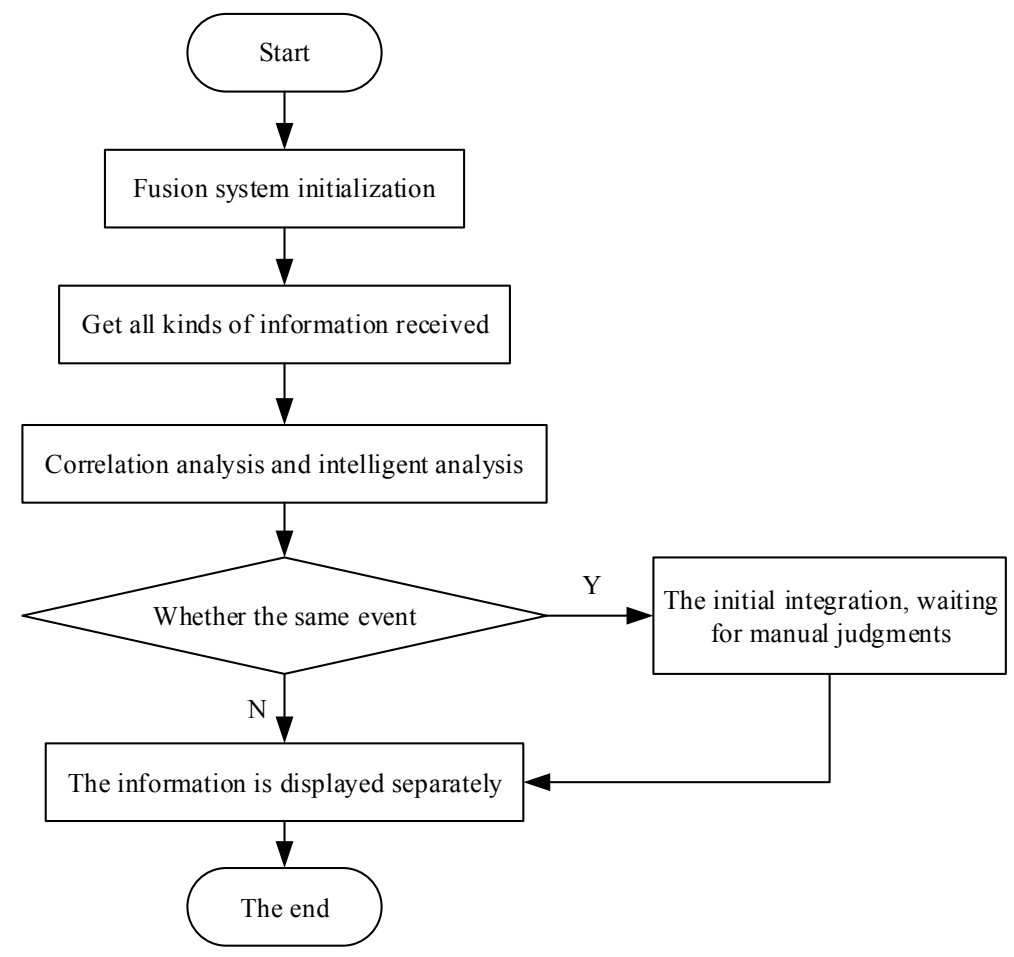

Fig. 5. Electronic patrol function software flow chart 


\section{$4 \quad$ Experiment}

Based on smart park information system of Internet of things and cloud computing, the intelligent security system is selected as an example to solve the problem of timely interconnection of information departments. It can be said that it is a typical intelligent interconnection park information system.

The experiment selects the video server based on cloud computing to realize the effective monitoring of the abnormal behavior in the park. We select Acro Tetra digital cluster single carrier base station SB421 as outdoor base station, and select SepuraSRH3800, and it integrates the world's leading GPS module. SepumsRH3800's positioning sensitivity is $-152 \mathrm{dbm}$, tracking sensitivity is $-155 \mathrm{dbm}$, and it can faster and easier precede locating than the average GPS receiver even among the buildings. It also passed the drop and vibration test of ETs300019Drop \& Vibration, and it's waterproof and dustproof value meet IEc529IP54 standard, storage temperature range is $-40^{\circ} \mathrm{C} \sim 85^{\circ} \mathrm{C}$, the ambient temperature range is $-20^{\circ} \mathrm{C} \sim 60^{\circ} \mathrm{C}$, and it can reach the low temperature storage and use. We select AcroUlliteU7200 as integrated communication platform, which is a new type of communication system. It can integrate wired and wireless communications, analog communication and digital communications, TDM and IP and other communications networks into a seamless network, in order to achieve interconnection between the various networks and unified command and dispatch.

The practicality picture of SepuraSRH3800 is shown in Figure 6.

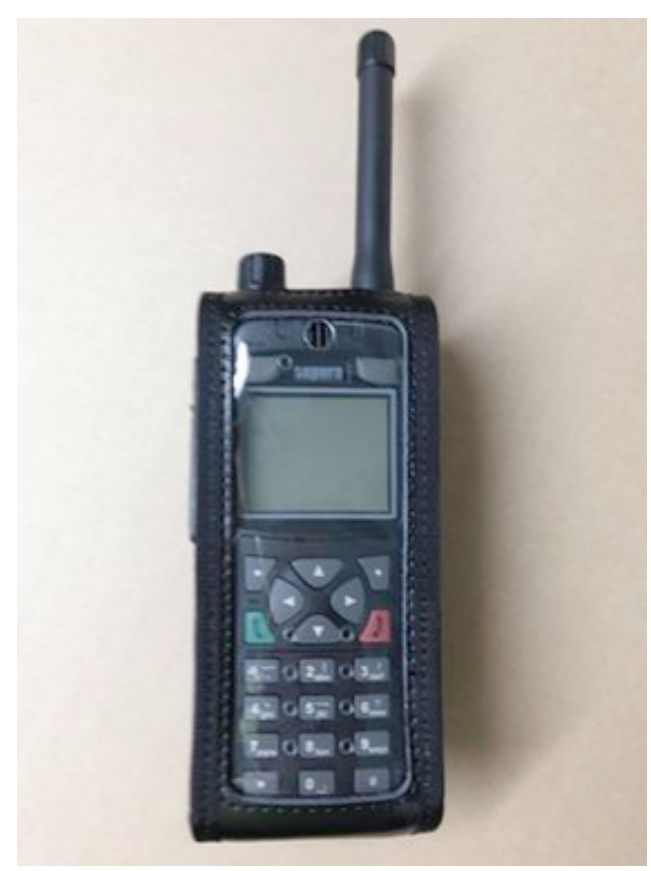

Fig. 6. The practicality picture of SepuraSRH3800 


\subsection{Experimental procedure}

This experiment mainly has three sections; the first section is cluster terminal positioning display. The specific method of this part is to numbering three sepurasRH3800 digital cluster hand-held terminals as A, B, C. Then we store the park map in the intelligent security system, when we receive location information sent by handheld terminal A, B, C, we could compare it with the actual position of $\mathrm{A}, \mathrm{B}, \mathrm{C}$. The second part is staff gathered alarm and fusion processing distribution, and the specific method is to enclose the monitoring areas, set the maximum number of people intensive in intelligent security system. This experiment set to 15 , when the actual number larger than 15, intelligent security system will give the alarm tips, obtain traffic information and environment information of control points, generate comprehensive information after the integration process, distribute to the terminal of relevant staff, and test the accuracy of system according to actual situation. The third part is the interconnection of PSTN and cluster hand-held terminal, mobile. And PSTN interconnect the cluster hand-held terminal A, B, C based on schedule dialing. Cluster D interconnect with digital cluster terminals A, B, C. Cluster D interconnect with mobile E.

\subsection{Experimental results and discussion}

We experiment 10 times for cluster terminal positioning, and record the distance between positioning and actual location of each time.

The implementation interface for cluster terminal location is shown in Figure 7. The results are shown in Figure 8.

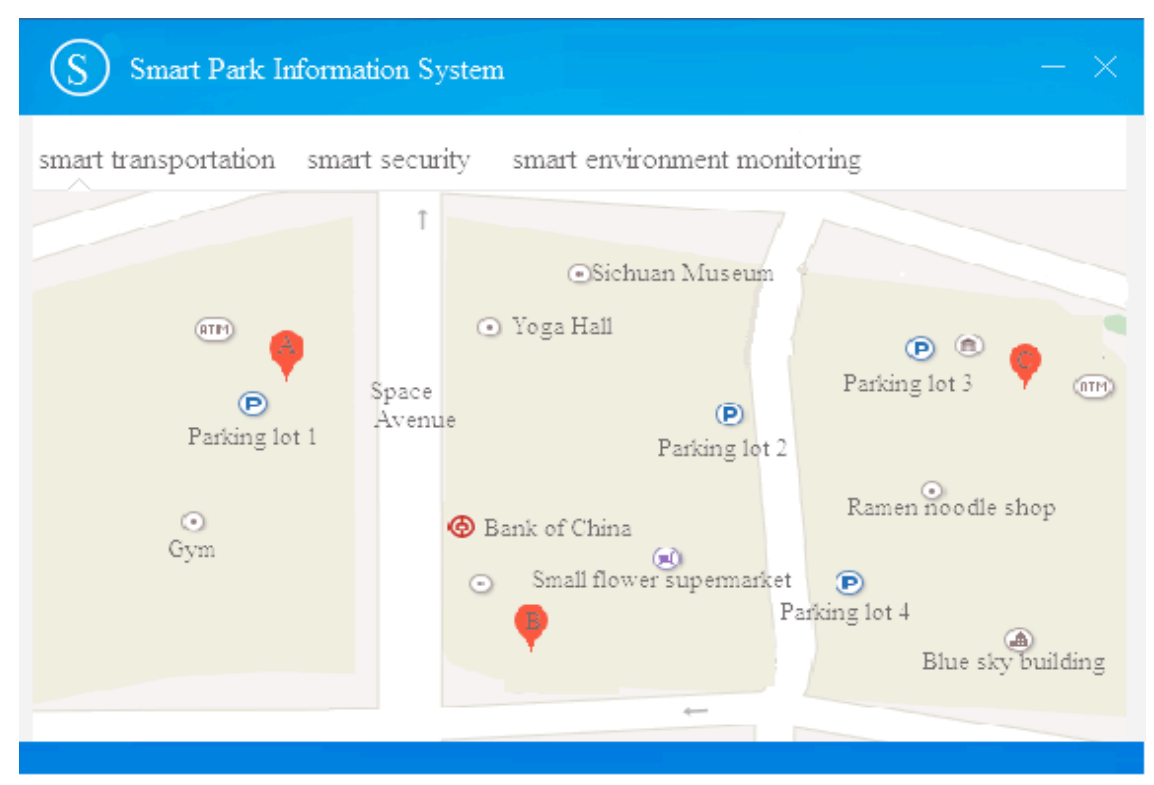

Fig. 7. The implementation interface for cluster terminal location 


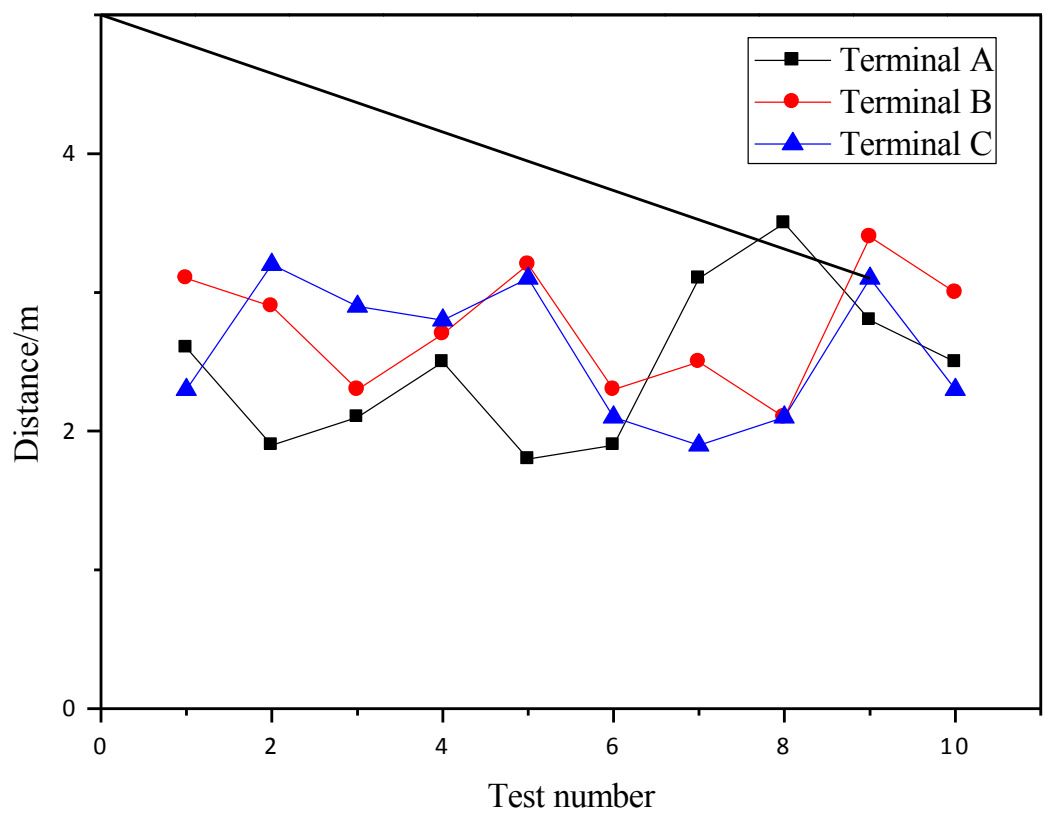

Fig. 8. Cluster terminal positioning experiment result chart

It can be seen from Figure $6, \mathrm{~A}, \mathrm{~B}, \mathrm{C}$ cluster terminal positioning error within 4 meters and it indicates the accuracy of system positioning is high, thereby meet the daily needs of smart park.

In the experiment of personnel gathering alarm and fusion processing distribution, when the number of people in video more than 15 , the intelligent security system will automatically give an alarm, and report the alarm information (location and number of aggregation limit) to the intelligent integration system. The experiment were carried out 10 times in the park at different locations, the experimental results are shown in

Table 1. System function test results

\begin{tabular}{|c|c|c|c|}
\hline Test number & $\begin{array}{c}\text { The number of people } \\
\text { in the video }\end{array}$ & Actual number & System feedback \\
\hline 1 & 13 & 14 & no-alarm \\
\hline 2 & 16 & 16 & alarm \\
\hline 3 & 14 & 16 & no-alarm \\
\hline 4 & 14 & 14 & no-alarm \\
\hline 5 & 15 & 15 & alarm \\
\hline 6 & 16 & 17 & alarm \\
\hline 7 & 17 & 17 & alarm \\
\hline 8 & 14 & 14 & no-alarm \\
\hline 9 & 13 & 14 & no-alarm \\
\hline 10 & 12 & 14 & \\
\hline
\end{tabular}


As we can be seen from Table 2, when the number of people in the video more than 15 , the system will automatically send an alarm. But in some situations, the video angle is not incomplete, and all people unable appear in the same video screen. But now, this system has met the daily needs of smart park.

PSTN interconnect digital cluster hand-held terminals A, B, C by dispatch station dialing, and the voice is clarity. Cluster $\mathrm{D}$ interconnect digital cluster terminals $\mathrm{A}, \mathrm{B}$, $\mathrm{C}$, and the voice is clarity. Cluster D interconnect designated mobile phone, and the voice is clarity. Therefore, this system can achieve timely and effective communication of emergencies.

\section{Conclusion}

The Internet of Things technology is rapidly developed, and cloud computing is gradually improved, which can be used as an effective support of information construction of smart park. The smart park has extensible ability, it introduces Internet of Things and other new technologies and advanced management tools under park operation stable premise, enhance social and economic benefits of smart park, and provide an effective reference for the development of smart city.

Smart park is an important manifestation and part of smart city construction. This study realizes the effective coordination of the intelligent systems in the park, eliminates the information island, satisfies the individual needs of the users in the park, expands the connotation of the smart park in the Internet of Things era, and has reference significance and application value to smart park and smart city.

\section{References}

[1] Bakıcı, T., Almirall, E., \& Wareham, J. (2013). A smart city initiative: the case of Barcelona. Journal of the Knowledge Economy, 4(2), 135-148. https://doi.org/10.1007/s13132012-0084-9

[2] Centenaro, M., Vangelista, L., Zanella, A., \& Zorzi, M. (2016). Long-range communications in unlicensed bands: the rising stars in the iot and smart city scenarios. IEEE Transactions on Vehicular Technology, 23(12), 5850-5865.

[3] Kitchin, R. (2014). The real-time city? Big data and smart urbanism. GeoJournal, 79(1), 114. https://doi.org/10.1007/s10708-013-9516-8

[4] Lombardi, P., Giordano, S., Farouh, H., \& Yousef, W. (2012). Modelling the smart city performance. Innovation: The European Journal of Social Science Research, 25(2), 137149. https://doi.org/10.1080/13511610.2012.660325

[5] Neirotti, P., Marco, A. D., Cagliano, A. C., Mangano, G., \& Scorrano, F. (2014). Current trends in smart city initiatives: some stylised facts. Cities, 38(5), 25-36. https://doi.org/10.1016/j.cities.2013.12.010

[6] Paroutis, S., Bennett, M., \& Heracleous, L. (2014). A strategic view on smart city technology: the case of ibm smarter cities during a recession. Technological Forecasting \& Social Change, 89, 262-272. https://doi.org/10.1016/j.techfore.2013.08.041

[7] Petrolo, R., \& Mitton, N. (2014). Towards a smart city based on cloud of things. (pp.6166). ACM. https://doi.org/10.1145/2633661.2633667 
[8] S., R., \& Roche, S. (2014). Geographic information science i: why does a smart city need to be spatially enabled?. Progress in Human Geography, 38(5), 315-7.

\section{$7 \quad$ Author}

Meiyan Du is with the Department of General Education, Shandong University of Arts, Shandong, China (gbothj589153@126.com).

Article submitted 04 April 2017. Published as resubmitted by the author 12 May 2017. 